Published in final edited form as:

Org Lett. 2005 June 9; 7(12): 2505-2508. doi:10.1021/ol050900q.

\title{
Superacid-Catalyzed Reactions of Olefinic Pyrazines: an Example of Anti-Markovnikov Addition Involving Superelectrophiles
}

Yiliang Zhang, Jason Briski, Yun Zhang ${ }^{\dagger}$, Rendy Rendy ${ }^{\dagger}$, and Douglas A. Klumpp

Department of Chemistry and Biochemistry, Northern Illinois University, DeKalb, Illinois 60115

tDepartment of Chemistry, California State Polytechnic University, Pomona, California, 91768

\section{Abstract}
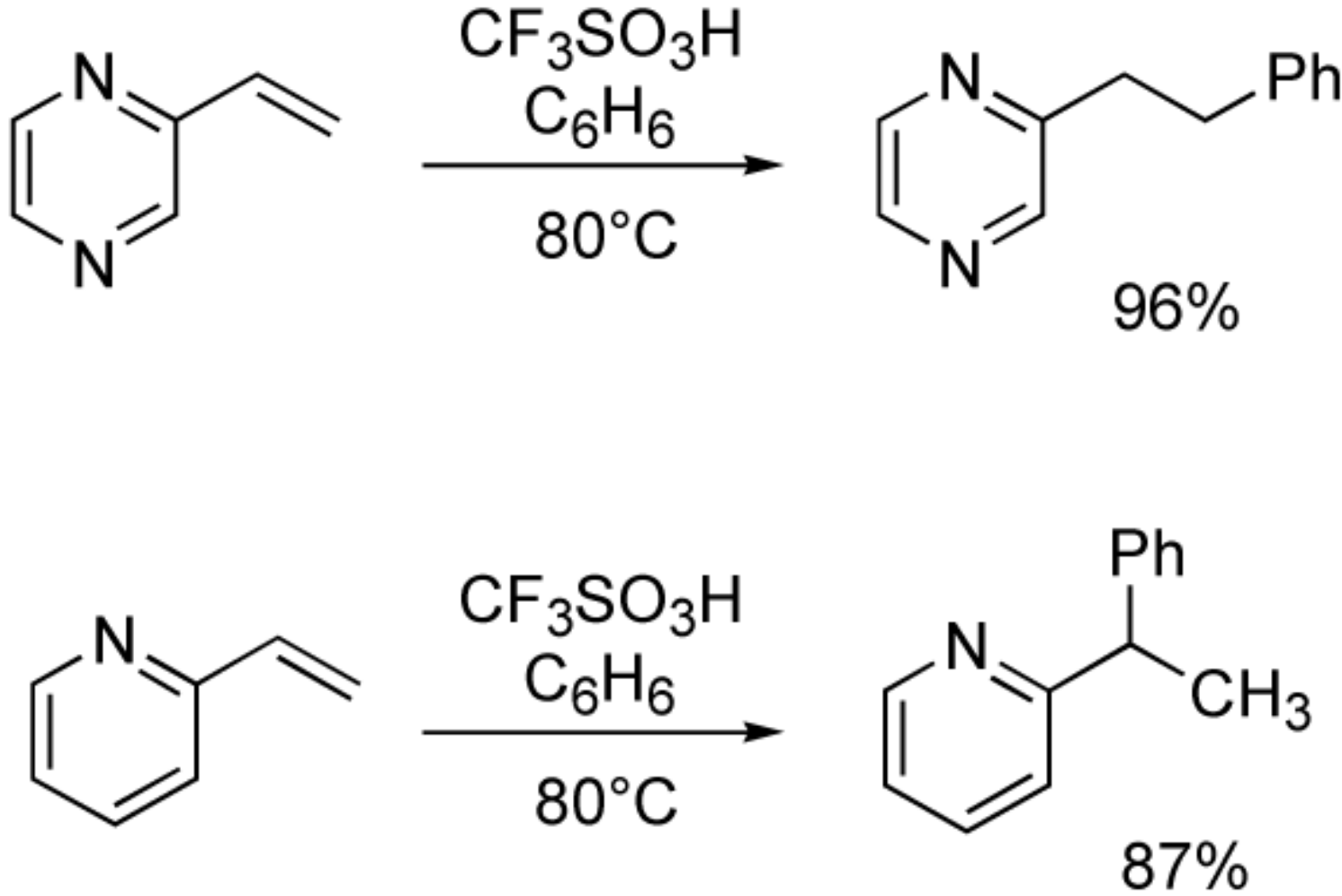

Olefinic pyrazines are found to react with benzene in $\mathrm{CF}_{3} \mathrm{SO}_{3} \mathrm{H}$ and give anti-Markovnikov-type addition products. We propose that this is caused by two effects: destabilization of the carbocationic intermediates that would lead to Markovnikov-type products and the generation of a considerable amount of positive charge at the terminal carbon of the olefinic groups. This suggests that acidcatalyzed addition reactions can give anti-Markovnikov-type products when a multiply charged (i.e. superelectrophilic) group is adjacent to the olefinic site. 
Superelectrophilic activation was first proposed by Olah and co-workers to explain the high reactivities of electrophilic species in superacidic media. ${ }^{1}$ When dissolved in superacids, electrophilic reagents like nitronium salts $\left(\mathrm{NO}_{2}{ }^{+} \mathrm{X}^{-}\right)$and acetylium salts $\left(\mathrm{CH}_{3} \mathrm{CO}^{+} \mathrm{X}^{-}\right)$are capable of reacting with exceptionally weak nucleophiles (i.e. alkanes and deactivated arenes). Despite having a formal positive charge, these electrophilic reagents can interact with the superacid through their non-bonding electron pairs and the superelectrophilic species are formed $\left(\mathrm{HNO}_{2}{ }^{2+}\right.$ and $\mathrm{CH}_{3} \mathrm{COH}^{2+}$ or partially protonated forms). There have been several recent studies in which olefinic superelectrophiles undergo Michael addition with very weak nucleophiles like benzene. ${ }^{2}$ In two of these studies, conjugate addition leads to products in which
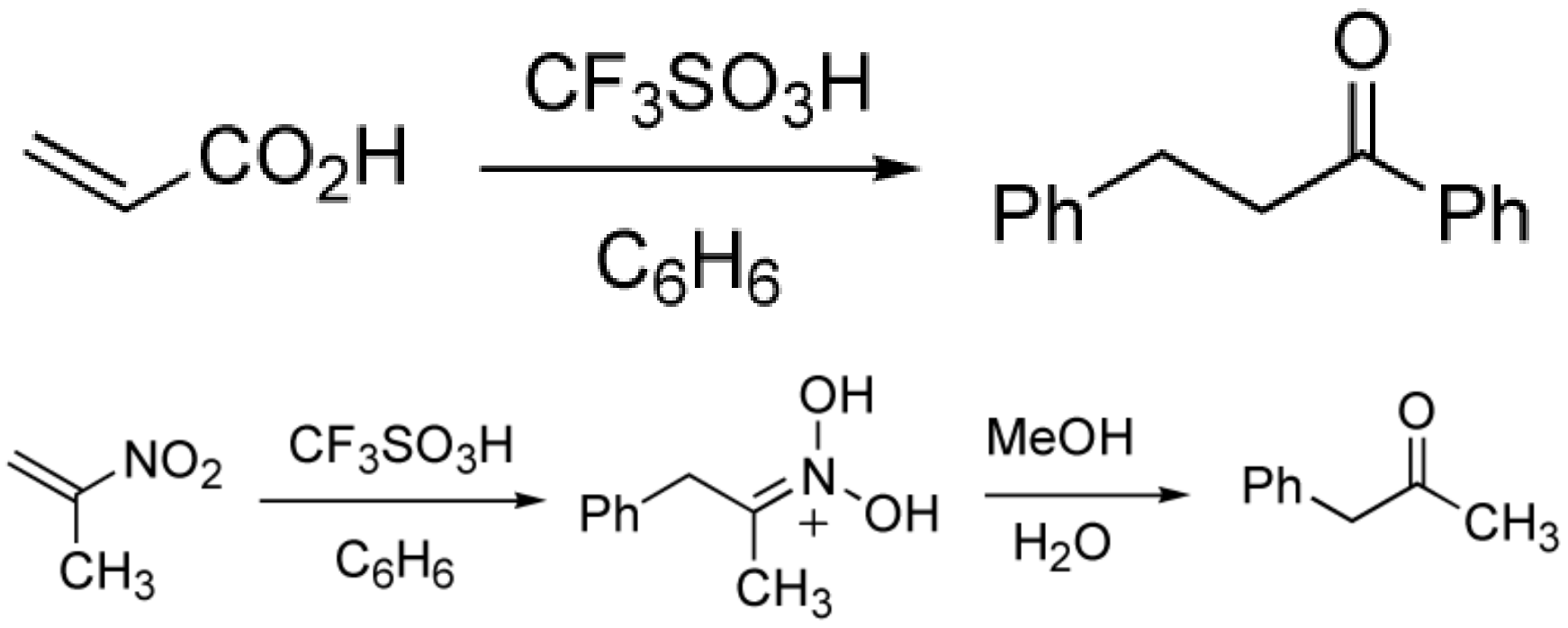

nucleophilic attack has occurred at the less-substituted position of an olefinic group (eq 1-2), leading to anti-Markovnikov addition in one case.2a,b Anti-Markovnikov addition chemistry has been of general interest since the concept was first proposed, and most known cases of anti-Markovnikov addition involve free-radical, ${ }^{3}$ photochemical, ${ }^{4}$ or transition metalpromoted reactions. ${ }^{5}$ In the following manuscript, we report the superacid-catalyzed reactions of olefinic pyrazines with benzene and the formation of anti-Markovnikov addition products. We also propose a general route towards anti-Markovnikov addition products involving olefinic superelectrophiles.

When vinylpyrazine $\left(\mathbf{1 a}, \mathrm{R}=\mathrm{H}\right.$ ) is reacted with the $\mathrm{Br} ø$ nsted superacid $\mathrm{CF}_{3} \mathrm{SO}_{3} \mathrm{H}$ (triflic acid) and benzene, the anti-Markovnikov product (2a) is formed as the only major product (Scheme 1). In contrast, 2-vinylpyridine, 1-vinylimidazole, and 5-vinylthiazole react under similar conditions to give exclusively the (Markovnikov) addition products having the 1-phenylethyl group (eq 3). ${ }^{6}$ Remarkably, the 2 -isopropenyl and the $\alpha$-sytryl substituted pyrazines ( $\mathbf{1 b}$ and 1c) likewise give the anti-Markovnikov addition products ( $\mathbf{2 b}$ and $\mathbf{2 c}$, respectively). Although protonation at the terminal carbon of compound $\mathbf{1 c}$ would generate a benzylic carbocation center and lead to Markovnikov addition, compound $\mathbf{2 c}$ is the only major product.

In order to further probe this chemistry, an isotopically labelled derivative (3) was prepared and reacted with $\mathrm{CF}_{3} \mathrm{SO}_{3} \mathrm{H}$ and $\mathrm{C}_{6} \mathrm{H}_{6}$ (eq 4). Analysis of the product (4) reveals that the ${ }^{13} \mathrm{C}$ label is only at the benzylic position. When 2-isopropenyl 
<smiles>C=Cc1ccccn1</smiles>
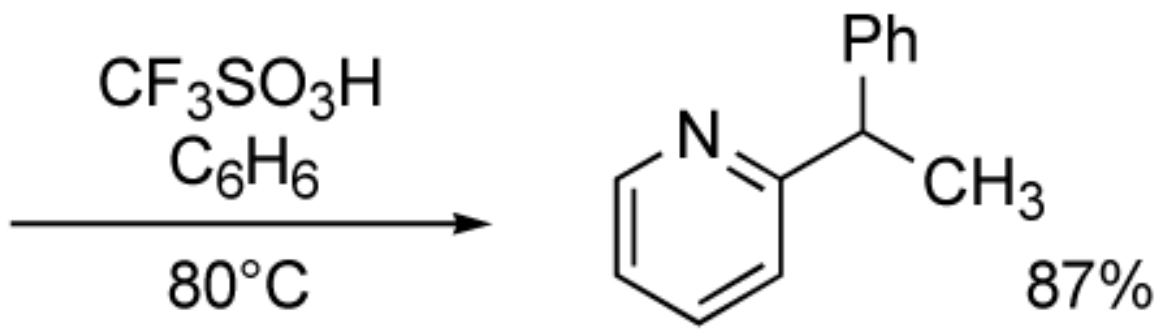<smiles>C=C(C)c1cnccn1</smiles>
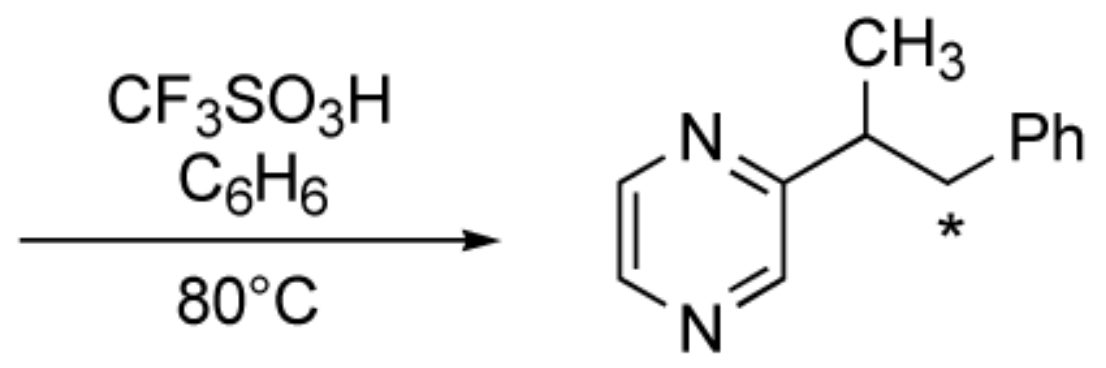

3

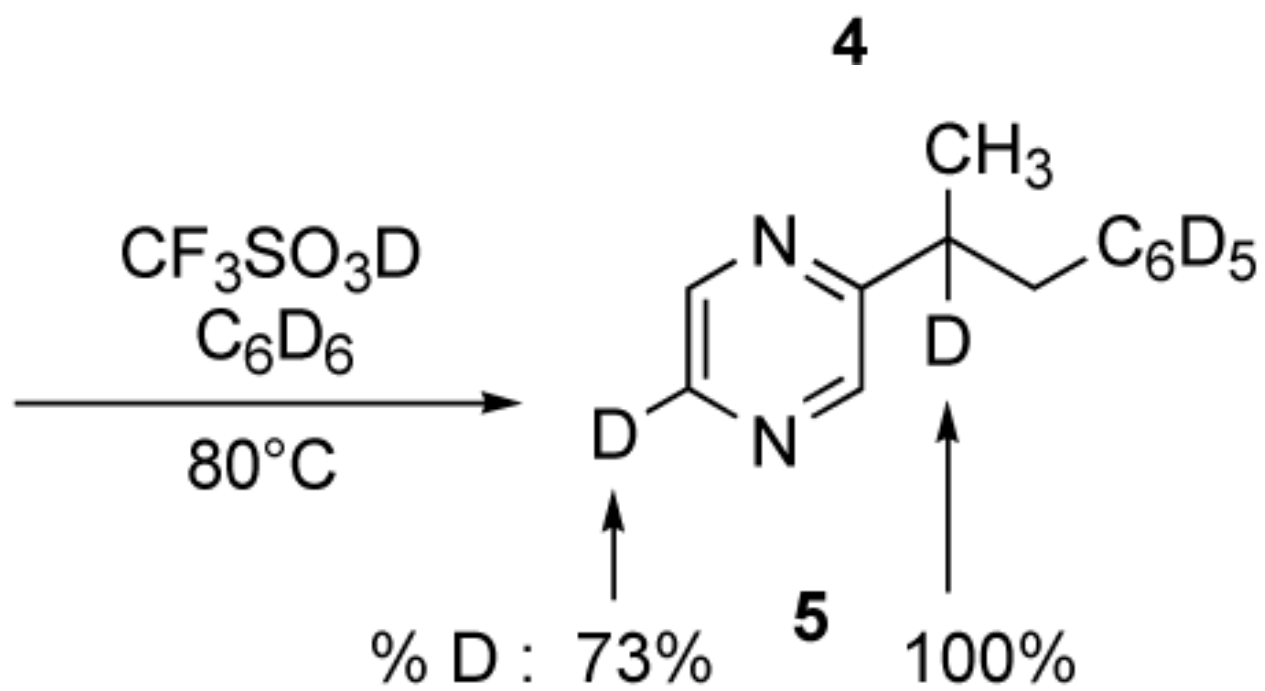

pyrazine (1b) is reacted with $\mathrm{CF}_{3} \mathrm{SO}_{3} \mathrm{D}$ and $\mathrm{C}_{6} \mathrm{D}_{6}$, the product 5 is found to have deuterium incorporation at the methine carbon and on the pyrazine ring at the 5-position (as well as on the phenyl ring; eq 5). ${ }^{7}$ Using alcohol substrates as precursors to cationic intermediates, the pyrazines give both the expected substitution products as well as rearrangement products (Scheme 2). In the case of the 1-hydroxyethyl pyrazine (6a), the substitution product (7a) is formed exclusively, while the methyl and phenyl-substituted pyrazines $(\mathbf{6 b}-\mathbf{c})$ give mixtures of the possible products. If product $7 \mathbf{c}$ is isolated and redissolved in $\mathrm{CF}_{3} \mathrm{SO}_{3} \mathrm{H}$ and $\mathrm{C}_{6} \mathrm{H}_{6}$, it does not isomerize to product $\mathbf{2 c}$.

The above results are consistent with the formation of tricationic species involving diprotonated and triprotonated pyrazines. The $\mathrm{pK}_{\mathrm{a}}$ values for methylpyrazine have been estimated to be 1.45 and $-5.25,{ }^{8}$ so it is reasonable to assume that the olefinic pyrazines are completely diprotonated in the excess superacid $\left(\mathrm{CF}_{3} \mathrm{SO}_{3} \mathrm{H},{ }^{9} \mathrm{H}_{0}-14.1\right)$. In order for deuterium incorporation to occur in the reactions of $\mathbf{1 b}$ with $\mathrm{CF}_{3} \mathrm{SO}_{3} \mathrm{D}$, the pyrazine must be deuterated at the two nitrogens and at a ring carbon (forming a trication), since deuteration at just one ring nitrogen and a ring carbon is highly unlikely. This suggests a reaction mechanism in which the diprotonated 
olefinic pyrazines (8a-c, Scheme 3) are in equilibrium with a ring-protonated tricationic species. Triprotonation of the pyrazine rings generate the superelectrophiles $(\mathbf{9 a - c})$ and this leads to the formation of positive charge on the less-substituted position of the olefinic groups. The incoming nucleophile $\left(\mathrm{C}_{6} \mathrm{H}_{6}\right)$ then reacts at the less-substituted position and subsequent protonation gives the anti-Markovnikov addition products (2a-c). In the addition reaction between $\mathbf{1 b}$ and $\mathrm{CF}_{3} \mathrm{SO}_{3} \mathrm{D}$ with $\mathrm{C}_{6} \mathrm{D}_{6}$ (eq 5), there is incomplete deuterium incorporation onto the pyrazine ring, suggesting that the addition reaction is occuring at a faster rate than protonation of the pyrazine ring. This leaves open the possibility of an alternative mechanism involving protosolvation of the olefin group with concominent nucleophilic attack by the arene nucleophile, an $\mathrm{Ad}_{\mathrm{E}} 3$ type mechanism (eq 6). ${ }^{10}$ Deuterium incorporation could then occur in a secondary reaction at the
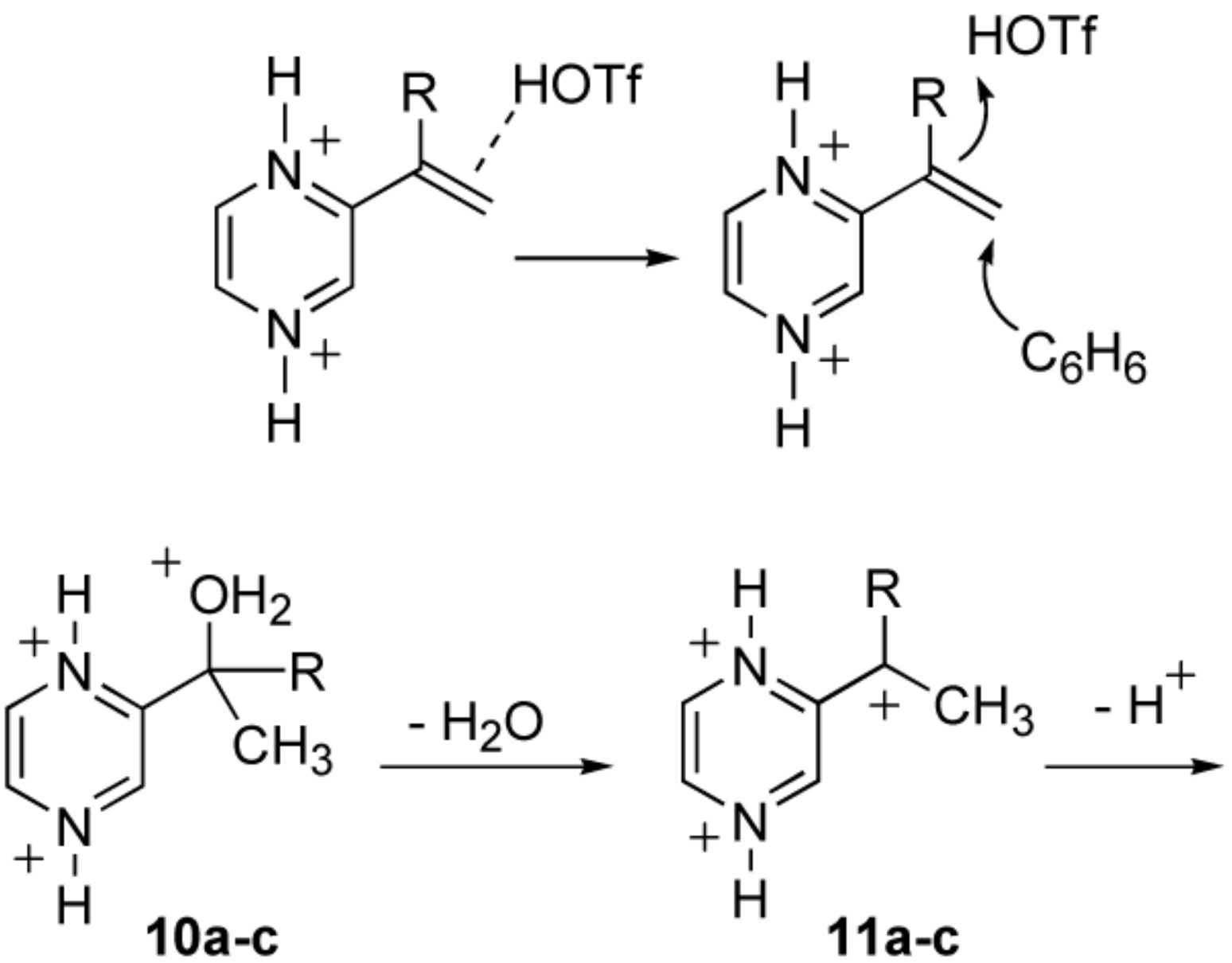

pyrazine ring. The alcohol substrates give two types of products: direct substitution products (7a-c) and the addition-type products (2a-c; Scheme 2). These results can be explained by assuming that the pyrazine rings are doubly protonated and an oxonium ion is formed by protonation of the hydroxy group (eq 7). The resulting trications (10a-c) can either undergo direct nucleophilic attack by benzene, or dehydration leading to a highly unstable carbocation (11a-c). Deprotonation then gives intermediates 8a-c leading to the anti-Markovnikov addition products. Despite having the favorable resonance stabilization, the phenyl-substituted carbocation (11c) rapidly undergoes deprotonation leading to the olefin $(\mathbf{8 c})$ and the addition product $(\mathbf{2 c})$. This suggests that the doubly-protonated pyrazine ring exerts a powerful 
destabilizing effect on the adjacent carbocationic center. We believe that this is the basis for the anti-Markovnikov addition involving the olefinic pyrazines.

There has been a recent suggestion that some reactions of superelectrophiles may occur by single electron transfer (SET) pathways, based on the results of quantum mechanical calculations. ${ }^{11}$ These computational studies showed that the lowest unoccupied molecular orbitals (LUMOs) of several dicationic electrophilies were energetically below that of the highest occupied molecular orbitals (HOMOs) of benzene and cyclohexane. Free radical chemistry is well known to produce anti-Markovnikov addition products, so to determine if radical cations are involved in the chemistry of the vinyl pyrazines, CIDNP experiments were done. For example, 4-(3-phenylpropyl)pyridine (12) was reacted with vinylpyrazine (1a) in triflic acid (eq 8) as a completely homogenous liquid phase. ${ }^{12}$ When the reaction is followed by ${ }^{1} \mathrm{H}$ NMR at $25^{\circ} \mathrm{C}$, no CIDNP signal enhancements or absorptions are observed. The reaction between $\mathbf{1 2}$ and $\mathbf{1 a}$ however, gives the expected<smiles>C=Cc1cnccn1</smiles>

addition product (13) in good yield. While the failure to observe CIDNP effects cannot rigorously exclude the possiblity of SET mechanisms and radical intermediates, it should be noted that a SET mechanism between a trication (like 9a) and the protonated form of $\mathbf{1 2}$ would produce a pair of radical dications from a SET pathway. Although dimerizations of radical cations have been reported previously, ${ }^{13}$ there are no known examples of dimerizations involving radical dications. The present results suggest that long-lived radical intermediates are not present in the reaction of $\mathbf{1 a}$ and $\mathbf{1 2}$ and consequently SET mechanisms are not involved in the reactions of the olefinic pyrazines (1a-c).

Based on the preliminary results described above, it is clear that the doubly charged pyrazine ring plays an important role in the protonation equilibria and the regiochemistry of nucleophilic attack. Moreover, this chemistry provides further evidence that superelectrophilic activation can be the basis for Michael addition leading to anti-Markovnikov-type products. When the earlier studies involving acrylic acid and

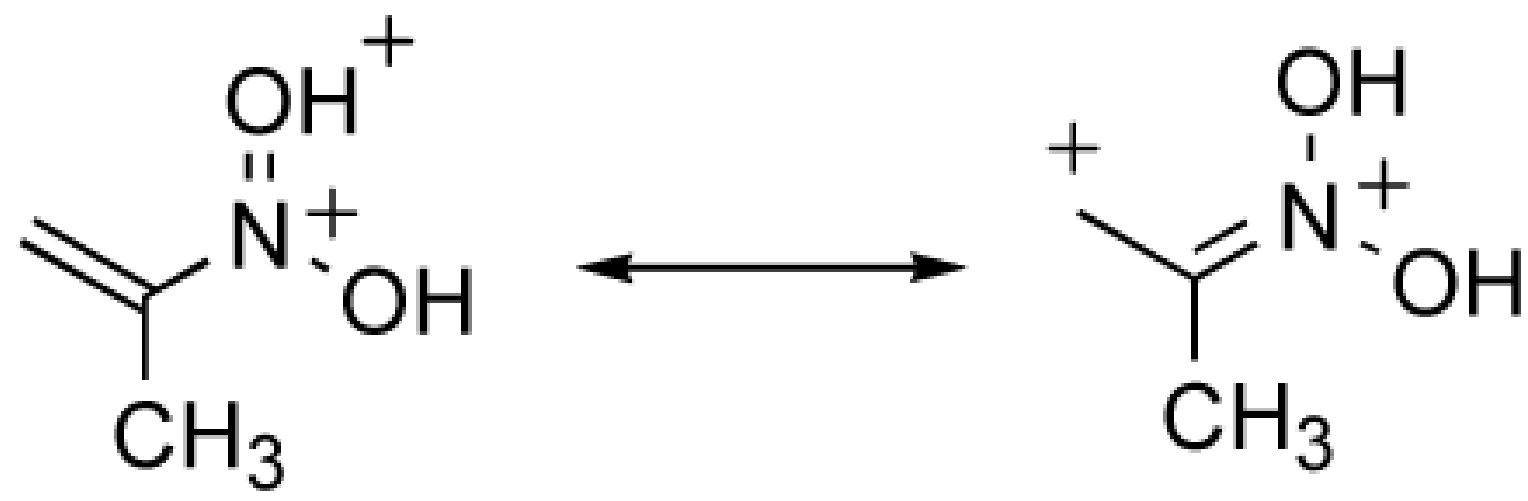



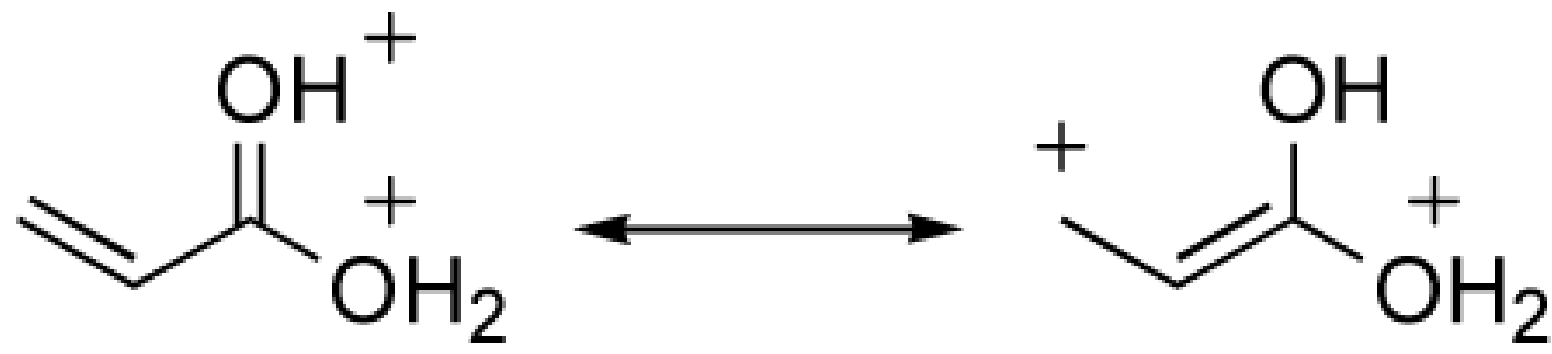

2-nitropropene are also considered, these systems all have a considerable amount of positive charge generated at the terminal (less-substituted) carbon (eq 9-10). ${ }^{14}$ This causes nucleophilic attack to occur at the terminal carbon leading to formal anti-Markovnikov addition in the case of acrylic acid.

In summary, olefinic pyrazines are found to react with benzene in $\mathrm{CF}_{3} \mathrm{SO}_{3} \mathrm{H}$ and give antiMarkovnikov-type addition products. We propose that this is caused by two effects: destabilization of the carbocationic intermediates that would lead to Markovnikov-type products and the generation of a considerable amount of positive charge at the terminal carbon of the olefinic groups. This suggests that acid-catalyzed addition reactions can give antiMarkovnikov-type products when a multiply charged (i.e. superelectrophilic) group is adjacent to the olefinic site. Not only can superelectrophilic activation enable chemistry with very weak nucleophiles, but our results show that it can also be the basis for unusual regiochemistry. Further studies are in progress to explore the scope of this type electrophilic activation and Michael addition.

\section{Supplementary Material}

Refer to Web version on PubMed Central for supplementary material.

\section{Acknowledgments}

The financial support of the NIH-NIGMS (GM071368-01 and SO6GM53933-0251), Merck-UNCF, and Northern Illinois University is greatly appreciated.

Supporting Information Available: Experimental procedures and characterization data, inlcuding ${ }^{1} \mathrm{H}$ and ${ }^{13} \mathrm{C}$ NMR data spectra and low and high resolution mass spectra data. This material is available free of charge via the Internet at http://pubs.acs.org.

\section{References}

1. (a) Olah GA. Angew. Chem., Int. Ed. Engl 1993;32:767. (b) Olah GA, Klumpp DA. Acct. Chem. Res. 2004

2. (a) Prakash GKS, Yan P, Torok B, Olah GA. Catal. Lett 2003;87(3-4):109. (b) Ohwada T, Okabe K, Ohta T, Shudo K. Tetrahedron 1990;46:7539. (c) Klumpp DA, Rendy R, Zhang Y, Gomez A, McElrea A. Org. Lett 2004;6:1789. [PubMed: 15151415] (d) Rendy R, Zhang Y, McElrea A, Gomez A, Klumpp DA. J. Org. Chem 2004;69:2340. [PubMed: 15049628]

3. Neumann R, de la Vera F, Bar-On A. J. Org. Chem 1995;60:1315.

4. Kojima M, Ishada A, Kuriyama Y, Wada Y, Takeya H. Bull. Chem. Soc. Jpn 1999;72:1049.

5. Beller M, Seayad J, Tillack A, Jiao H. Angew. Chem., Int. Ed 2004;43:3368. and references cited therewithin.

6. Zhang Y, McElrea A, Sanchez GV Jr. Klumpp DA, Do D, Gomez A, Aguirre SL, Rendy R, Klumpp DA. J. Org. Chem 2003;68:5119. [PubMed: 12816465] 
7. Assignment of the regiochemistry of deuteration was based on ${ }^{1} \mathrm{H} N M R,{ }^{13} \mathrm{C}$ NMR, HETCOR, and mass spectral data, see Supporting Information. Further comparisons were made with published NMR data of an alkylpyrazine, see: Cox RH, Bothner-By AA. J. Phys. Chem 1968;72:1646.

8. Chia AS-C, Trimble RF Jr. J. Phys. Chem 1961;65:863.

9. Olah, GA.; Prakash, GKS.; Sommer, J. Superacids. Wiley; New York, NY: 1985.

10. (a) Fahey RC, Monahan MW. J. Am. Chem. Soc 1970;92:2816. (b) Emery SL, Fies CH, Hester EJ, McClusky JV. J. Org. Chem 1999;64:3788.

11. Koltunov KY, Prakash GKS, Rasul G, Olah GA. J. Org. Chem 2002;67:8943. [PubMed: 12467412]

12. With benzene as a substrate, two phases separate: the acidic-ionic phase and the nonpolar benzene phase.

13. (a) Masui M, Ueda C, Moriguchi T, Michida T, Kataoka M, Ohmori H. Chem. Pharm. Bull 1984;32 (4):1392. (b) Park JW, Choi NH, Kim JH. J. Phys. Chem 1996;100:769. (c) Apperloo JJ, Groenendaal LB, Verheyen H, Jayakannan M, Jayakannan M, Janssen RAJ, Dkhissi A, Beljonne D, Lazzaroni R, Bredas J-L. Chem. Eur. J 2002;8:2384.

14. Although no mechnaism was specifically proposed for the reaction of acrylic acid with benzene in $\mathrm{CF}_{3} \mathrm{SO}_{3} \mathrm{H}$ (ref. 2a), the $O, O$-diprotonated intermediate is perhaps the most likely dicationic species based on earlier studies involving carboxylic acids in superacidic media. See: Prakash GKS, Rasul G, Burrichter A, Laali KK, Olah GA. J. Org. Chem 1996;61:9253. 


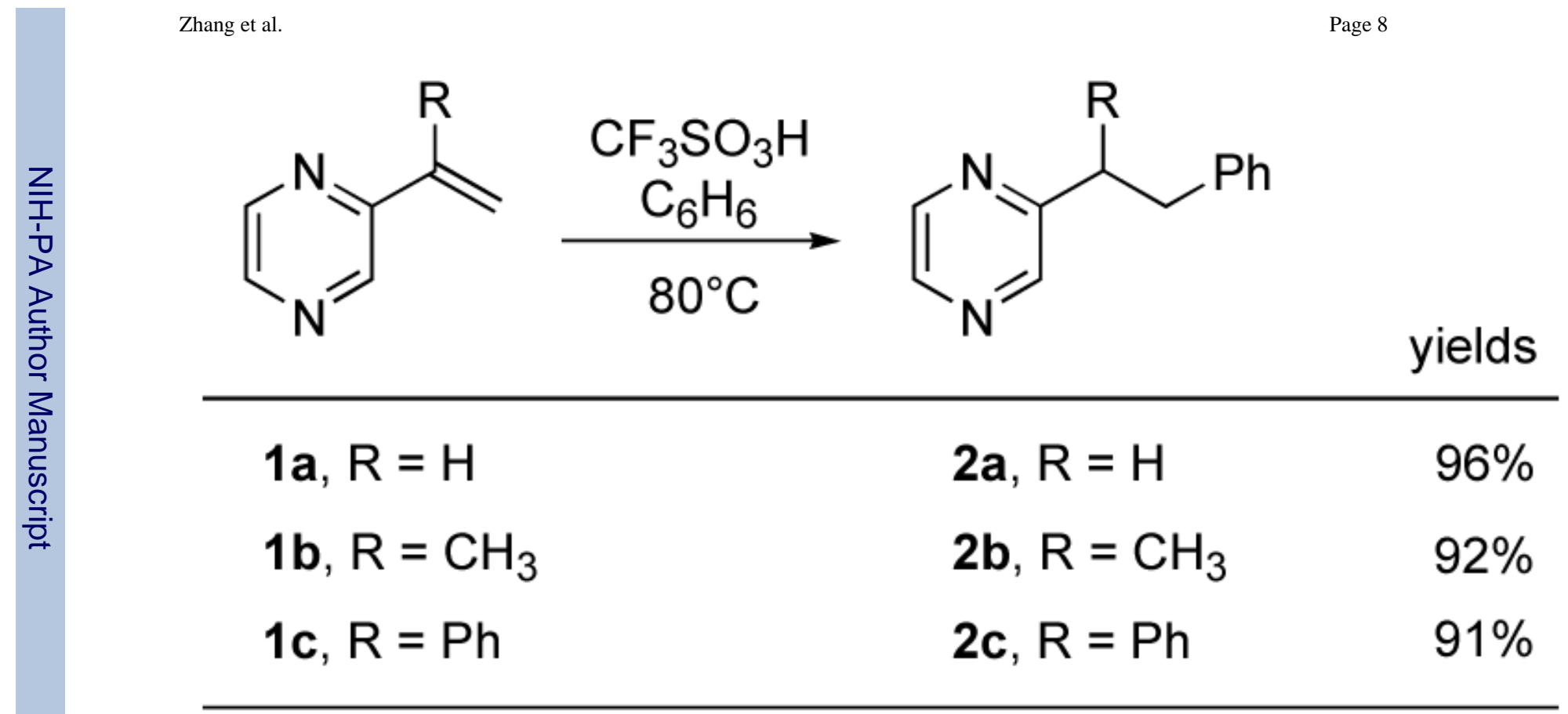

Scheme 1.

Reactions of olefinic pyrazines. 


Zhang etal.
6a, $\mathrm{R}=\mathrm{H}$
$\mathbf{6 b}, \mathrm{R}=\mathrm{CH}$
$\mathbf{6 c}, \mathrm{R}=\mathrm{Ph}$

Scheme 2.

Reactions of alcohols 6a-c. 
<smiles>[R]C(=C)c1cnccn1</smiles><smiles>[R]C(=C)C1=[NH+]C[CH+]C([R3])[NH+]=C1</smiles>

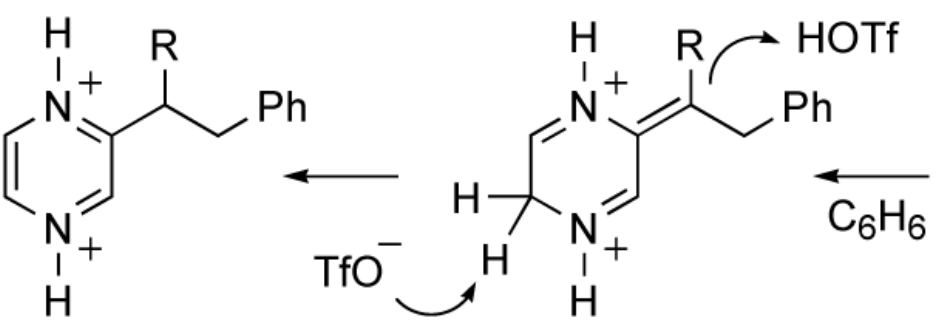<smiles></smiles>

Scheme 3.

Proposed mechanism of addition. 